\title{
Traduire le roman africain francophone en slovène
}

\author{
Katja Zakrajšek \\ Traductrice indépendante
}

\begin{abstract}
C'est pour des motifs politiques et religieux plus sérieux qu'il [le roi] arrêta les cours [de français]. Il connaissait plus que tout autre l'arbitraire des commandants. Maintenir un interprète entre le Blanc et lui, c'était se réserver une distance, quelques libertés, un temps de réflexion, des possibilités de réticences et de commentaires ; entretenir une certaine incompréhension. (Kourouma, Monnè 225-226)
\end{abstract}

Dans son roman Monnè, outrages et défis, Kourouma explore les thèmes du dialogue interculturel/interlinguistique, des disparités des codes linguistiques et systèmes de références, des malentendus qui en résultent et des possibilités de manipulation stratégique de ces malentendus. Ce champ thématique s'organise autour du personnage de l'interprète : au moment de l'irruption coloniale dans l'ordre politique, social et culturel établi, il assume un rôle fondamental, extrêmement ambivalent, de l'intermédiaire entre «le peuple du Soba » (ou plutôt son chef) et le conquérant français.

Loin d'être une innocente "médiation interculturelle ", la traduction constitue ici une médiation entre des positions de pouvoir et d'intérêt asymétriques, ce qui lui confère inévitablement une forte charge politique. Cette évocation du caractère complexe et difficile du processus de la traduction nous servira de point de départ pour une réflexion sur la traduction de littératures africaines europhones.

Le présent article $^{1}$ se concentre notamment sur la traduction dans des langues « mineures » européennes, qui présente quelques spécificités notables par rapport à la traduction dans des langues dites «majeures ». Il s'agit d'une précision importante dans la mesure où la critique traductologique semble dominée par les questions concernant la médiation vers ces dernières. Bien que les auteurs associés aux tournants culturel et postcolonial en traductologie soient très attentifs aux dissymétries de pouvoir entre langues, leur réflexion, axée le plus souvent sur la traduction vers l'anglais, n'est pas directement applicable aux situations où la langue cible se trouve en position plus faible par rapport à la langue source. Afin de combler cette lacune théorique, je propose quelques observations en me fondant en partie sur ma propre expérience de traduction de textes africains francophones en slovène. Les exemples concrets (peu nombreux pour des raisons pratiques) se réfèrent donc à cette langue.

Je pars de la position que la traduction littéraire devrait préserver et communiquer au lecteur cible l'intérêt littéraire du texte source, ou, comme l'a dit K. A. Appiah, reprenant la définition barthesienne de la littérature comme « ce qui s'enseigne »: " une bonne traduction d'un texte littéraire devrait préserver les caractéristiques grâce auxquelles il mérite d'être enseigné » (398). Dans cette perspective, la traduction littéraire est indissociable d'une analyse littéraire, implicite ou explicite, du texte source, ou plus exactement, analyse et traduction s'éclairent

\footnotetext{
${ }^{1}$ Je tiens à remercier les évaluateurs de cet article pour leur précieux commentaires et suggestions qui m’ont aidé à en clarifier certains aspects. - Je reprends ici, en partie, l'argumentation que j’ai développée dans deux articles publiés en slovène: « $\mathrm{K}$ problematiki prevajanja afriškega evrofonskega romana $\mathrm{v}$ slovenščino » [Considérations sur la traduction du roman africain europhone en slovène] et «Potujitveni in prisvajajoči prevod v luči medjezikovnih razmerij moči » [Défamiliarisation et assimilation dans la traduction dans la perspective des relations de pouvoir entre langues] (voir bibliographie).
} 
mutuellement ${ }^{2}$. Je commence donc par esquisser une approche théorique de textes africains francophones avant d'aborder le sujet de leur traduction.

\section{Littératures africaines, langues européennes}

À la différence des littératures européennes, les littératures africaines europhones emploient des langues exogènes imposées par la colonisation et qui restent toujours des langues dominantes ou élitaires (à norme exogène, notamment européenne), parlées (encore moins lues) par une minorité de la population. En Afrique, l'ancienne langue coloniale contribue toujours à une hétéroglossie déjà complexe, où elle coexiste avec une ou plusieurs langues endogènes et très souvent aussi avec une des ses variétés locales, non standardisées (par exemple avec le pidgin nigérian, le camfranglais, le français d'Abidjan, etc,) qui sont en train de devenir des langues véhiculaires des milieux urbains. La position des langues européennes en Afrique est donc très différente de celle en Europe, où la langue nationale coïncide avec la langue maternelle et la langue d'écriture, et où le discours sur la pratique littéraire présuppose souvent, tacitement, que cette situation représente l'état naturel des choses.

En réfléchissant sur la production littéraire europhone en Afrique (ainsi que sur certaines autres littératures europhones non-occidentales), il convient donc de prendre en compte les stratégies possibles que peuvent adopter les écrivains pour se situer par rapport aux anciennes langues coloniales et/ou pour se les approprier. Au niveau déclaratif, l'on peut repérer toute une gamme de prises de position, de la déclaration fameuse de Tchicaya U Tam'si: «la langue française me colonise, je la colonise à mon tour » (Gauvin 41), en passant par la naturalisation stratégique de la langue européenne, par exemple, chez Abdourahman A. Waberi : « je connais la langue et la littérature française depuis le berceau scolaire» (Martin et Drevet 107), à la position exprimée par Kossi Efoui : «Je pense que n’importe quelle langue convient [...] Parce que le point de départ de l'écriture, c'est un acte de méfiance, vis à vis de n'importe quelle langue, y compris la sienne propre» (Martin et Drevet 127). Au niveau de la production des textes littéraires, la situation est encore plus complexe, ambivalente et souvent paradoxale, puisque le texte normalement s'adresse à des publics très différents (africains et non-africains) aux horizons de lecture divergents. Un texte positionné ainsi dans un entre-deux culturel qui d'ailleurs s'y trouve souvent explicitement thématisé, ne peut être réduit par la traduction à un seul système de références ou à l'homogénéité de la culture cible sans perdre une grande partie de sa signifiance et de son intérêt.

Dans les littératures africaines europhones, il n'y a généralement pas de coïncidence entre la langue d'écriture et la langue (les langues) de la réalité sociale à laquelle cette écriture se réfêre. Confrontés à des pratiques langagières, à des représentations et normes linguistiques plurielles qui s'insèrent dans des relations assymétriques de pouvoir symbolique et politique, les écrivains opèrent des médiations complexes entre ce contexte original et la langue d'écriture. La textualisation de l'hétéroglossie sociale est autant un problème technique et esthétique qu'un problème politique. Cela a des conséquences directes pour la traduction: plus les stratégies linguistiques d'un tel texte sont chargées d'implications politiques, moins les choix traductifs sont innocents. Comme nous verrons plus tard (voir partie 2.1), dans le cas de la traduction vers les

\footnotetext{
2 Paul Bandia procède de manière comparable dans son ouvrage Translation as Reparation: sa réflexion sur la traduction de textes africains s'appuie sur l'analyse de ceux-ci (leur politique langagière, stratégies de représentation culturelle, etc.). Bandia insiste sur l'écriture interculturelle en tant que traduction, soulignant les procédés textuels qui permettent d'inclure d'autres langues dans la langue d'écriture. Mon approche est légèrement différente en ce que j'analyse tant les stratégies d'écriture que de traduction dans la perspective bakhtinienne de l'image de langage (au niveau de l'écriture, cela inclut les cas d' "écriture comme traduction », mais aussi la représentation de variétés de la langue d'écriture).
} 
langues «mineures » européennes qui nous intéresse ici, ce problème est particulièrement difficile à résoudre en raison des exigences contradictoires auxquelles la traduction est censée répondre dans ces conditions.

En même temps, c'est un problème que le traducteur ne peut éviter, puisque la politique culturelle des textes francophones africains se manifeste très souvent au niveau du traitement de la langue (ou des langues); loin d'être un simple ornement stylistique, les procédés d'écriture qui en découlent constituent un élément essentiel de la signification du texte. Mon analyse s'appuie sur la stylistique du roman proposée par Mikhail Bakhtine. Pour ce dernier, le roman est un genre hybride qui «a toujours évolué à la lisière des cultures et des langues» (Esthétique 410); ses particularités seraient « organiquement liées et conditionnées par une certaine crise dans l'histoire des sociétés européennes » qui débouche sur une prise de conscience des autres langues et cultures: «L'époque de coexistence des langues nationales en circuit fermé est terminée. Les langues s'éclairent mutuellement, car une langue ne peut être consciente d'elle-même qu'à la lumière d'une autre. » (ibid. 448). Il est vrai que Bakhtine parle d'un moment précis de l'histoire européenne, mais ses propos sont très pertinents pour d'autres situations sociohistoriques où le monde est « devenu activement plurilingue une fois pour toutes » (ibid.), comme c'est le cas dans les contextes postcoloniaux qui nous intéressent ici. La conception bakhtinienne de la langue en tant que réseau dynamique de pratiques sociales et la stylistique du roman axée sur le traitement textuel de l'hétéroglossie sociale constituent un excellent point de départ pour aborder l'analyse de textes romanesques produits hors des contextes nationaux classiques du type « occidental». Les notions bakhtiniennes qui me semblent les plus utiles dans la réflexion sur les problèmes de traduction sont l'image de langage et l'orchestration. Dans ce qui suit, je propose une esquisse de différents types d'images de langage que l'on trouve dans les romans africains francophones avant d'aborder les questions proprement traductologiques.

\subsection{Image de langage et orchestration}

Le terme «image de langage » se réfère à la manière dont tel langage social - dialecte, registre, langage d'un courant littéraire, etc. - est représenté dans le roman : bref, la manière dont les discours préexistants y sont traités et stylisés. Selon Bakhtine, une telle représentation artistique est toujours dialogique et bivocale, parce qu'il s'agit d'une rencontre entre au moins deux langages sociaux qui sont de véritables protagonistes du roman polyphonique. Leurs rapports peuvent varier: des éléments de l'un peuvent s'infiltrer dans l'autre au sein d'une seule unité syntaxique, ou bien la voix représentatrice « accentue de façon particulière (humoristique, ironique, parodique etc.) » (Bakhtine Esthétique 119) le discours social représenté. Ce qui importe est qu'il n’y a pas de lignes de démarcation nettes entre ces différentes évaluations (ou «accentuations»): la parole bivocale est, de par sa nature même, un site d'ambivalence. Elle est rendue possible par la mise à distance qui résulte de la prise de conscience de la prolifération de discours divergents, contradictoires au sein de la langue.

Bien que Bakhtine ait dévéloppé sa théorie à partir du corpus des "grands » romans européens, cette insistance sur la prise de conscience d'autres voix (de voix des autres) et d'autres perspectives, avec toutes les conséquences éthiques et politiques qui en découlent, fait que sa pensée se prête bien aux contextes liminaires, pluriels, au sein desquels évoluent les littératures africaines europhones. La conception bakhtinienne de la langue, où la norme centrale n'est pas privilégiée mais vue comme un discours social parmi d'autres, permet de décrire toute hétéroglossie locale dans sa pluralité et sa spécificité, qu'il s'agisse de situations où se rencontrent plusieurs langues ou qui se limitent à une seule langue "nationale ». Les notions d'image et de stylisation font se déplacer le centre de l'attention des questions de "correction ", de "fidélité » ou d'«authenticité » (Est-ce vraiment cela que dirait un locuteur camérounien?) vers celles 
d'efficacité (Est-ce une représentation convaincante, évocatrice, du français camérounien?) ${ }^{3}$. C’est une perspective qui s'avère intéressante également pour l'analyse de choix traductifs.

Pour Bakhtine, le style du roman équivaut au traitement textuel de l'hétéroglossie sociale: l'on pourrait le décrire comme une configuration textuelle d'images (représentations) artistiques de langages sociaux, une "diversité littérairement organisée » (Bakhtine Esthétique 88). "Grâce à ce plurilinguisme et à la plurivocalité qui en est issue, le roman orchestre tous ses thèmes, tout son univers signifiant, représenté et exprimé. » (ibid. 89) Il est vrai que le terme bakhtinien d'« hétéroglossie » (" plurilinguisme » dans le passage cité) est peu rigoureux et peut renvoyer tant aux situations linguistiques réelles qu'à la coprésence de langues dans le texte littéraire. Afin d'éviter ce type de confusion, R. Grutman a proposé le terme " hétérolinguisme » pour désigner «la présence dans un texte d'idiomes étrangers, sous quelque forme que ce soit, aussi bien que de variétés (sociales, régionales ou chronologiques) de la langue principale » (37). Enfin, il serait utile de disposer aussi d'un terme qui se réfèrerait plus particulièrement aux stratégies d'organisation de cet hétérolinguisme, stratégies qui participent à la politique culturelle du texte. Le mot « orchestration » employé par Bakhtine lui-même (bien que non systématiquement), me parait particulièrement approprié : comme le remarque $M$. Holquist, il renvoie aux procédés de composition romanesque polyphonique (Bakhtine Dialogic 430-431), et cadre bien avec perception bakhtinienne du roman comme une partition musicale.

\subsection{Images de variétés du français non standard}

La notion d'image de langage est particulièrement utile dans le cas de pratiques langagières non standard, telles les variétés locales des anciennes langues coloniales en Afrique (français urbain) et les langues pidgin et créoles qui en sont issus; les unes et les autres se font de plus en plus présentes en littérature, surtout dans les littératures anglophones mais aussi lusophones et francophones. La notion bakhtinienne d'image artistique permet d'observer les stratégies textuelles d'évocation de ces pratiques langagières, plutôt que d'insister sur la question de leur «transposition» dans le code écrit standard. C'est une manière fructueuse d'aborder des références littéraires aux variétés "populaires » des (anciennes) langues impériales ou aux langues de contact non stabilisées.

Le roman Temps de chien de Patrice Nganang en est un bon exemple. Ici, des représentations du français populaire de Yaoundé et du camfranglais parsèment un texte qui se sert du français comme une matrice neutre permettant la communication dans un contexte africain international. En 2001, date de la parution du roman, ce français paraît déjà vidé de références à l'ancien centre colonial et redéfini en tant que langue africaine. L'on en trouve une illustration remarquable dans les notes ironiques en bas de page dans la première édition du roman, où l'auteur a fourni des traductions de quelques expressions en camfranglais (selon toute probabilité à la demande de son éditeur français) : pour ces clarifications, Nganang ne s'est pas servi du français européen standard, mais bien du français urbain d'Afrique. Ainsi la phrase «If he no fit chop he moni, n'est-ce pas la mbok-là va l'aider? » est traduite par "S'il ne pouvait pas manger son argent, n'est-ce pas la pute-là va l'y aider » (Lefebvre 41).

\footnotetext{
${ }^{3}$ Les pratiques langagières en marges des langues standard et dans les régions de contact de langues ne connaissent pas, en général, la forme écrite, encore moins une forme écrite stabilisée. Cela fait que l’inclusion de ces langages sociaux marginaux dans la littérature écrite est toujours une question technique tout autant que de politique culturelle: en pratique, l'écrivain se trouve confronté à la nécessité que son texte soit (relativement) lisible. Mais lisible pour quel lecteur? Africain? Européen? Au reste, que veut dire "lisible": immédiatement accessible ou exigeant éventuellement une participation active du lecteur?
} 
Cet procédé met bien en lumière la résistance de nombreux auteurs à l'idée de créer une (illusion de) transparence afin de faciliter (ou fausser?) la compréhension (et pour quel lecteur ${ }^{4}$ ?). Le problème semble d'ailleurs se poser d'une manière plus aiguë en Afrique francophone qu'en Afrique anglophone; en effet, il semble que la décentralisation linguistique relative des espaces anglophones (par contraste au poids de la norme parisienne) a facilité la production et la réception de textes expérimentant avec les variétés linguistiques locales, par exemple le pidgin nigérian. Ici, l'enjeu est bien la légitimité de ces pratiques, leur « droit » à accéder à la langue écrite et particulièrement à la littérature. Pour le moment, leur présence dans le roman africain francophone se limite généralement au dialogue ; la traduction du roman Sozaboy, où les traducteurs ont représenté l'image du pidgin nigérian proposée par Saro Wiwa en construisant un langage qui se fonde sur le français d'Abidjan, constitue pourtant une expérience extrêmement intéressante où la subversion du français standard par un code local concerne l'intégralité du texte et illustre les possibilités de ce type d'écriture en français .

\subsection{Images de langues africaines}

La notion d'image de langage est également utile dans les cas où une langue et une culture sont exprimées dans une autre langue, dominante, qui ne constitue pourtant pas un élément de l'hétéroglossie locale présentée par le texte. Dans ces conditions, je propose de distinguer, pour les besoins de la traduction, entre langue de départ (généralement la langue maternelle de l'écrivain) et langue d'écriture, ou langue source.

Les écrivains africains francophones ont dévéloppé plusieurs procédés de représentation de langues africaines en français. Certains ont recours à la transposition de structures syntaxiques et (plus souvent) de certains genres de discours (allant des formules de salutation aux proverbes etc.), produisant ainsi un texte original déjà-traduit où la langue de départ, présente en palimpseste, transforme la langue d'écriture. En français, l'exemple le plus connu et le plus analysé est sans doute celui de Les Soleils des indépendances d'Ahmadou Kourouma. Ce roman est bien écrit en français (langue source) mais la langue de ses protagonistes et (au moins en partie) de son narrateur est le malinké (langue de départ). Ici, la parole bivocale dont parle Bakhtine se fait donc trivocale, puisque le français langue littéraire se superpose au métissage variable des voix malinkées du narrateur et des protagonistes :

Là, entre les toits, apparaissaient divers cieux : le tourmenté par les vents qui arrachaient des nuages pour les jeter sur le soleil déjà couvert et éteint, le bas épais et indigo montant de la mer et avançant sur les maisons et les arbres inquiets et tremblotants. L'orage était proche. Ville sale et gluante de pluies! pourrie de pluies! (Kourouma Soleils 21)

Dans ce passage, les singularités morphosyntaxiques (substantivation insolite des adjectifs) sont attribuables au discours du narrateur en malinké, auquel se joint, dans la dernière phrase, la parole du personnage principal Fama. L'écrivain, ancien colonisé, s'est arrogé le droit de s'approprier la langue dominante, de la démonter et de la réorganiser afin d'en faire un outil pour la représentation de discours culturels que la langue coloniale avait étouffés ou niés. L'image de la langue colonisée dans la langue coloniale (ou plus largement, de la langue dominée dans la langue dominante), la subordination du texte en langue coloniale à la langue colonisée - et non seulement dans les dialogues, où cela pourrait fonctionner comme un simple élément de couleur locale, mais bien dans le discours du narrateur comme c'est le cas dans Les Soleils - voilà une stratégie à forte charge politique, inséparable de l'effet esthétique du texte.

\footnotetext{
${ }^{4}$ Je me concentre ici sur le point de vue du lecteur européen, étant donné le sujet principal de cet article.
} 
D'autres auteurs parsèment leurs textes de mots ou d'expressions en langue de départ, qu'ils peuvent soit traduire ou expliquer dans le texte même ou en notes en bas de page (solution très commune dans les textes moins récents), soit laisser au lecteur la tâche d'en déduire la signification à partir du contexte (cf. Zabus, op.cit.). Certains voient ces expressions non traduites comme des représentants symboliques de la culture de départ et de ses aspects les plus importants. Pourtant, il est plus fructueux de les considérer comme des métonymes, des évocations de la différence culturelle structurant le texte, souvent écrit dans un régistre standard ou même soutenu de la langue source.

\subsection{Représentations textuelles de plurilinguismes extratextuels}

Enfin, la notion d'image de langage est utile pour l'analyse des textes où une réalité radicalement plurilingue est comme "aplanie" pour former une surface textuelle plus ou moins monolingue. Le plurilinguisme du milieu socioculturel de référence constitue un problème technique autrement plus difficile pour l'écrit que pour l'audivisuel (rappelons les différents traitements de la langue parlée par les personnages dans le roman et le film Xala de Sembène Ousmane). Pourtant, cette situation peut donner lieu à des effets très intéressants.

L'Étrange destin de Wangrin en est un bon exemple : dans ce texte, écrit tout en français à l'exception de quelques emprunts translittérés, l'on trouve les images du français standard, du «français tirailleur» et de plusieurs langues ouest-africaines endogènes. L'un des aspects linguistiques les plus intéressants de ce roman est le style soutenu, volontiers «littéraire», dans certains dialogues entre des locuteurs de langues africaines : «Ô ma sœur Rammaye, je me retiens pour te plaire, mais apprends que l'amour est comme un fleuve. Son eau roule, s'écoule et passe, mais jamais il ne tarit ni ne varie d'état. » (Hampaté Bâ 127). Pour le lecteur européen, cette association d'un haut registre du français et de l'imaginaire et de la rhétorique des langues ouestafricaines produit un double effet de familiarité et d'étrangeté radicale. Le haut régistre du français, qui évoque la culture dominante et sa tradition élitaire, y est récupéré pour représenter la culture marginalisée et son raffinement nié : l'appropriation et l'expropriation transgressives de la langue dominante opèrent un retournement éloquent des positions de pouvoir et de savoir, puisque le discours des personnages français est rendu dans un langage beaucoup plus ordinaire.

\section{Stratégies de traduction}

Nous pouvons maintenant reformuler le problème de la traduction pour y inclure les considérations mises en relief par l'analyse bakhtinienne : comment représenter, dans la langue cible et avec les moyens offerts par celle-ci, le texte source avec son orchestration d'images de langages plurielles et sa manière de traiter l'hétéroglossie sociale - bref : sa politique linguistique et culturelle? C'est une question de principe qui ne concerne pas exclusivement la traduction des littératures africaines europhones. Le défi particulier que posent les romans africains francophones (et plus généralement europhones), textes excentrés aux systèmes de références culturelles et aux points d'entrée multiples, est le suivant : il n'est guère possible de trouver une solution adéquate dans les limites ordinaires d'une langue cible (nationale). Pour des raisons historiques, il est très rare de trouver dans la langue cible une configuration exactement correspondante de langages sociaux que l'on pourrait utiliser dans la traduction pour réactualiser la politique culturelle du texte dans le (super)système cible. En ce qui concerne les littératures ouest-africaines, il existe une correspondance assez étroite entre le français et l'anglais (et à la rigueur, le portugais) quant à leur sociologie et le rapport entre leurs variétés locales et la variété standard dominante (ce qu'ont exploité avec bonheur les traducteurs en français du roman Sozaboy). Ce n'est pas le cas des autres langues européennes. En outre, à la différence de la 
traduction entre le français et l'anglais, dans la traduction du français vers une langue "mineure », tel le slovène, une asymétrie de pouvoir entre les langues source et cible vient s'ajouter à celle entre les composantes de l’hétérolinguisme du texte source.

\subsection{Le tournant postcolonial en traductologie et les " petites" langues européennes}

Les auteurs associés aux tournants culturel et postcolonial en traductologie - L. Venuti, G. Spivak, H. Bhabha et autres -, ont repensé la traduction dans la perspective de relations de pouvoir (symbolique ou réel) entre les langues source et cible. Toutefois, ils se concentrent sur la traduction vers l'anglais, langue dominante par excellence. Pour cette raison leurs conclusions, pourtant très intéressantes, ne sont pas directement applicables aux situations de traduction où la langue cible est en position plus faible (marginale, dominée) par rapport à la langue source. Par exemple, tandis que la critique de la traduction cibliste par L. Venuti est certainement valable pour l'anglais, N. Pokorn rappelle que certaines cultures mineures « ont constitué et renforcé leur littératures » grâce à la traduction cibliste (des grandes littératures), " ce qui n'a certainement pas renforcé les tendances hégémoniques des grandes littératures » (153). Il s'agit donc d'élargir cette réflexion très fructueuse en tenant compte de ces autres situations de traduction, où l'appropriation (ou «domestication ») joue un rôle important en tant que stratégie de résistance antihégémonique.

La remarque précédente vaut aussi pour la réflexion sur la traduction des littératures africaines, qui se limite généralement aux échanges entre les deux grandes anciennes langues coloniales, l'anglais et le français (voir p. ex. Translation as Reparation de Paul Bandia et Decoloniz̨ing Translation de Kathryn Batchelor). Dans le cas qui m'intéresse ici, la position traductive est plus complexe. D'une part, il s'agit de la traduction d'une langue «majeure » vers une langue « mineure »; d'autre part, de la traduction d'une position culturelle marginale vers une littérature nationale. Pour cette raison, le traducteur se trouve devant des exigences contradictoires, justifiées, toutes les deux, par la politique de résistance aux ethnocentrismes réducteurs qu'épouse la traductologie postcoloniale : d'un côté, le besoin pour la langue «mineure » et sa littérature de résister, dans une certaine mesure, à l'influence hégémonique d'une culture plus forte, afin de préserver leur intégrité ${ }^{5}$; d'un autre côté, l'exigence de représenter l'hétéroglossie qui structure le texte source postcolonial, les implications sociales de cette hétéroglossie ainsi que sa présentation artistique dans le texte original.

En théorie, ces deux objectifs sont parfaitement compatibles dans la mesure où il s'agit, dans les deux cas, de réponses concrètes à des asymétries de pouvoir culturel. En pratique, il n'est pourtant pas toujours facile de les concilier. D'une part, la traduction vers une langue "mineure » appelle une certaine appropriation et domestication linguistique du texte. D'autre part, pour représenter la subversion de la langue source, il faut soumettre la langue cible aux procédés comparables à ceux que subit la langue source dans l'original. Dans une certaine mesure, il est possible de concilier ces deux exigences par un traitement différencié des multiples composantes du texte, en limitant les procédés de domestication linguistique aux éléments qui relèvent du français standard. Dans la mesure où le slovène de la traduction représente le français hexagonal du texte original, il doit se plier aux variétés africaines du français ou aux langues endogènes afin

\footnotetext{
${ }^{5}$ Ce débat très intéressant meriterait plus d'attention que je ne peux y accorder ici. Je me limite à signaler simplement, à titre d'exemple, une statistique qui illustre l'importance de la traduction «coulante» (la «fluent translation» critiquée par Venuti) dans une langue comme le slovène : pour ne s'en tenir qu'au roman, selon le Service de statistique de la Slovénie (Statistični urad), 324 des 473 titres publiés en 2008 sont des traductions. Il est facile d'imaginer les conséquences pour la langue cible si toutes ces traductions s'ouvraient à l'altérité de leurs langues sources respectives (qui sont souvent des langues majeures, dont le français).
} 
de proposer leurs images au lecteur. Ainsi, la traduction d'un texte francophone africain fait jouer un rôle inédit au slovène, langue de l'Europe centrale qui n'a jamais été langue de colonisateur, mais qui, au contraire, avait elle-même été dans la position dominée et marginalisée dans l'empire austro-hongrois ; c'est une expérience de pensée aussi intéressante que difficile.

Il n'est guère possible, ni d'ailleurs souhaitable, de faire « disparaittre » une telle traduction en tant que traduction, selon l'idéal souvent formulé de traduction transparente, puisque les auteurs postcoloniaux mettent souvent intentionnellement en relief leur position historique spécifique et leur rapport complexe avec la langue exogène qui devient leur langue d'écriture, et que la politique culturelle des textes qui se manifeste dans les procédés d'écriture est un aspect essentiel de leurs œuvres. Cela va contre la pratique prédominante de la traduction cibliste, pourtant importante, on l'a dit, dans la traduction des grandes littératures (comme la littérature française) vers les petites littératures. Toutefois, une telle intervention postcoloniale ne constitue pas un désaveu des raisons en faveur de cette pratique prédominante, mais invite plutôt à une réflexion plus nuancée sur la position de la culture cible à l'égard de l'histoire et de l'état actuel des rapports de force à l'échelle mondiale. Afin de faciliter la réception d'une telle traduction transgressive d'une œuvre appartenant de surcroît à une littérature qui est très peu connue par le lectorat cible, il est utile de proposer au lecteur des informations sur le contexte historique et la situation linguistique textualisée dans le texte original, ainsi que des éclaircissements sur les choix de traduction. En outre, cet appareil paratextuel (préface ou postface de traducteur) aide le lecteur à retrouver dans le texte un autre culturel concret et bien défini, plutôt qu'une altérité abstraite sans ancrage sociohistorique.

\subsection{Allah n'est pas obligé et le français africain}

Dans le roman Allah n'est pas obligé, Ahmadou Kourouma a explicitement thématisé les tensions entre le français africain et la norme européenne, déjouant ainsi la tentation de les ramener aux termes de la langue cible (en recourant à des formes familières ou argotiques de celle-ci) ou bien à les neutraliser en « corrigeant» le texte:

Pour raconter ma vie de merde, de bordel de vie dans un parler approximatif, un français passable, pour ne pas mélanger les pédales dans les gros mots, je possède quatre dictionnaires. [...] Il faut expliquer parce que mon blablabla est à lire par toute sorte de gens : des toubabs (toubab signifie blanc) colons, des noirs indigènes sauvages d'Afrique et des francophones de tout gabarit (gabarit signifie genre). (Kourouma Allah 11)

Comment représenter une différence culturelle articulée au sein d'une même langue dans la langue cible où cette différence n'existe pas?

Afin de recréer cet aspect essentiel du roman, j'ai proposé un slovène africanisé hypothétique, en prenant en compte les transformations subies par le français dans le texte de Kourouma et les possibilités (et restrictions) offertes par la langue cible. À titre d'exemple, prenons une expression entièrement construite à partir du matériau lexical français : « faire pied la route" ('Inventaire des particularités lexicales du français en Afrique noire donne une expression légèrement différente, "prendre son pied la route ») : "Après ça nous n'avons pas encore fait longtemps pied la route, même pas dix kilomètres ... » (Kourouma Allah 46). Dans la traduction, la structure (verbe + nom + nom), anomale tant en français qu'en slovène, a été retenue et recréée en utilisant le matériau lexical slovène. Mais cette langue ne possédant pas de pro-verbe comparable au verbe "faire", très employé en français africain, j'ai dû utiliser le verbe iti («aller»; dans l'exemple proposé, la forme conjuguée est (ni)sva šla) : "Po tistem še nisva šla dolgo noga pot, niti deset kilometrov ...» (Kourouma Alabu 36). Ce procédé, qui permet de signaler l'intrusion d'un discours autre, a pourtant ses limites. Ainsi, il est impossible de recréer en slovène l'écart par rapport à la norme française que constitue le non-respect de la concordance des temps, typique de l'usage africain ("J'ai quitté le banc parce que tout le monde a dit que l'école ne vaut 
plus rien, » Kourouma Allah 9), puisque l'emploi des temps verbaux dans la langue cible correspond précisement à cet usage.

Le français africain n'étant qu'un aspect de la carnavalisation de la langue dans Allab n'est pas obligé, ces transformations de la langue cible se limitent à des passages isolés, bien qu'assez nombreux. Plus récemment, la bonne réception de la traduction en slovène d'un roman anglophone, Sozaboy, où l'image de la langue marginale (le pidgin nigérian) s'étend à l'ensemble du texte, a montré que ce procédé de «pidginisation » artificielle peut en principe être mis en œuvre à l'échelle du roman, sans que l'opacité intentionnelle d'une telle traduction repousse le lecteur ou nuise à la compréhension du texte.

\section{3 Éléments déjà traduits}

Par contraste à la situation décrite ci-dessus, l'hétérolinguisme textuel ne concerne pas des variétés standard et non standard d'une même langue, mais des langues différentes; il ne s'agit pas de représenter une pratique africaine du français, mais d'ouvrir le français aux langues autres (étouffées ou marginalisées par les politiques linguistiques coloniales et postcoloniales). Ici encore moins que dans le cas précédent, la traduction ne peut représenter ces effets textuels par des simples écarts de la norme de la langue cible; il faut ouvrir la langue cible au delà de l'hétéroglossie qui y existe déjà, en explorant l'espace du "possible adjacent», pour rendre possible une relation dialogique à l'autre sans le réduire aux termes de la culture cible.

Généralement, il n'est pas trop difficile de reconnaitre les images de la langue de départ et les transformations que celle-ci fait subir à la langue source (voir la distinction introduite en 1.3), à la condition toutefois que le traducteur se rende compte de cette possibilité et n'attribue pas ces effets stylistiques à la maladresse de l'auteur. Ces transformations peuvent ensuite être recréées dans la traduction dans un certaine mesure, d'autant plus facilement si la langue source et la langue cible partagent des caractéristiques morphosyntaxiques. Toutefois, il est très rare que le traducteur connaisse la langue de départ. Il est spécialiste du français, non africaniste, et généralement, l'image de la langue africaine dans la langue source, à savoir le français, est en effet sa source d'information principale et parfois unique sur cette langue africaine, ce qui peut limiter les choix de traduction.

Ahmadou Kourouma employait souvent des calques syntaxiques du malinké, comme dans cet exemple qui correspond à peu près à l'ablatif absolu en latin (Moro 361) : " Mais seul, quand Fama tournait ses longues nuits blanches, c'était lâchement apaisant » (Kourouma Soleils 127 ; c'est moi qui souligne). Tant la construction adjectivale détachée «mais seul » que l'emploi transitif, irrégulier du point de vue de la norme, du verbe «tourner» pourraient être recréés en slovène pour construire une phrase anomale mais pourtant compréhensible (ce qui correspond à l'effet de la phrase originale en français) : "Toda sam, ko je Fama premetaval svoje dolge nespečne noči, je bilo to podlo pomirjujoče».

Il est intéressant de noter qu'en comparaison avec le français, une langue comme le slovène, avec sa syntaxe souple, peut présenter des difficultés pour une telle stratégie, puisqu'il est plus difficile d'y recréer les effets d'étrangeté provenant d'un conflit entre la syntaxe de la langue de départ et celle de la langue source, surtout au niveau de l'ordre des mots dans la phrase. Néanmois, la structure, les conventions et l'imaginaire de toute langue européenne sont assez éloignés de ceux des langues ouest-africaines qui nous intéressent ici pour qu'il soit généralement possible de construire ces effets de défamiliarisation. 
La stratégie de transformation de la langue cible selon la logique de la langue de départ est plus facile à mettre en œuvre dans la traduction des métaphores et des genres de discours comme des proverbes, des formules de salutation, etc. En effet, l'emploi de ces éléments déjà traduits est aussi la stratégie la plus fréquente de l'inclusion en palimpseste de la langue de départ dans un texte écrit dans une langue autre. Ici, la traduction littérale peut s'avérer utile, avec la restriction toutefois que cette littéralité doit être réservée aux éléments qui résultent de l'influence de la langue de départ sur la langue source, et qu'il faut prendre en compte la structure de la langue cible.

Prenons la phrase très simple «Je suis là » par laquelle Rama répond à la question « Alors, Rama, comment vas-tu?» dans le roman Riwan (Bugul 84). C'est une traduction littérale de l'expression originale en wolof, maa ngi fi, dont l'équivalent français pragmatique serait «ça va » (une traduction mot-à-mot donnerait « je ici »; « je suis ici» en est la modification minimale pour former une phrase grammaticalement correcte en français). En slovène, l'équivalent de cette phrase ne serait donc ni «Jaz sem tukaj» (traduction mot-à-mot de la phrase source en français, à la rigueur grammaticale mais à connotations peu appropriées, correspondant plutôt à une phrase à pronom personnel tonique en français) ni «Jaz tukaj» (traduction mot-à-mot de la phrase originale en wolof, non grammaticale en slovène), mais "Tukaj sem, » solution qui concilie l'altérité de la convention dans la langue de départ et la structure grammaticale de la langue cible, ce qui correspond au texte source français.

\section{4 Éléments non traduits}

Enfin, la langue de départ peut se manifester par l'inclusion d'éléments non traduits dans le texte source. Dans les littératures africaines europhones, ces éléments sont donnés dans une translittération phonétique approximative, au début parce que la langue originale pouvait ne pas encore avoir de forme écrite en alphabet latin, et aujourd'hui peut-être aussi par habitude ou par convention. Quoi qu'il en soit, emprunter ces translittérations dans la traduction reviendrait à « coloniser» tant la culture de départ que la culture cible et les plier à la norme de la langue source (même sans tenir compte du fait qu'une telle transcription est inutile au lecteur cible). Afin d'éviter ce piège, nous avons donc deux possibilités : soit l'utilisation de l'orthographe de la langue de départ (si celle-ci possède une orthographe stabilisée), ce qui a l'avantage de présenter la langue de départ sans intervention d'une autre norme ; soit la translittération phonétique dans la langue cible, ce qui facilite la tâche de lecture au lecteur cible. Le choix dépend tant des considérations pratiques (par exemple : à quel point le traducteur a-t-il accès à l'information sur la langue de départ?) et des conventions dans la langue cible en ce qui concerne le traitement des emprunts. Ainsi, en traduisant Soundiata de Djibril Tamsir Niane, texte qui fait un usage abondant de mots, expressions et vers malinkés en transcription française, j'ai choisi d'en donner des translittérations en slovène, en accord avec l'auteur (communication personnelle) et avec l'aide aimable d'un locuteur du malinké, à commencer par le nom du héros qui devient «Sundžata » en slovène.

\section{Pour une politique de traduction non assimilatrice}

Les littératures africaines francophones présentent une série de défis de traduction qui nécessitent l'abandon du cadre conceptuel que constituent les notions de langue et littérature nationales; en revanche, les concepts bakhtiniens de l'image de langue et de l'orchestration de l'hétéroglossie s'avèrent très pertinents. Dans cette perspective, une traduction réussie d'un texte africain francophone est celle qui prend en compte la position culturelle (et politique) spécifique du texte et la présente au lecteur cible, non en traduisant des éléments de style isolés mais en reconstruisant leurs interrelations, soit en identifiant et réactualisant des discours sur l'autre/les 
autres présents dans la langue cible, soit en construisant des représentations de ces discours à partir de possibles de la langue cible. Un élément important d'une telle traduction non assimilatrice est l'appareil paratextuel qui fournit au lecteur des indications sur le contexte historique, culturel, politique et linguistique auquel se réfère et où s'insère le texte source, ainsi que sur les procédés de traduction.

Ce n'est pas qu'un enjeu stylistique; il s'agit d'une politique culturelle de traduction responsable qui évite d'occulter la spécificité de telle position (inter)culturelle particulière en y substituant une transparence illusoire ; au contraire, elle fait valoir le droit de l'autre à l'opacité, pour emprunter l'expression de Glissant. Ce n'est pas une médiation entre des essences culturelles bien délimitées, mais entre des positions culturelles fluides, plurielles et hybrides ; ou plus exactement, une représentation dans la langue cible de la représentation que le texte source construit de l'hétéroglossie de la réalité socioculturelle à laquelle il se réfère. Cela exige un certain effort tant de la part du traducteur que de la part du lecteur, qui se trouve obligé d'adopter une attitude active et ouverte face à l'autre (écrivant et écrit) représenté dans la traduction, et à accepter sa différence plutôt que de l'assimiler à un cadre de références préétabli. Le type de traduction que je viens de décrire aboutit à un texte plurivocal qui déstabilise les expectatives fondées sur la norme dominante et invite à la participation plutôt qu'à la consommation passive, tout en tenant compte de la spéficité sociohistorique tant du contexte de la production de l'œuvre que du contexte cible; par cela, elle s'inscrit dans le domaine de la "traduction postcoloniale » tout en l'élargissant. C'est en prolongeant la confrontation créative et souvent transgressive avec l'hétéroglossie sociale proposée dans le texte source qu'elle accomplit son projet d'activisme artistique et ouvre de nouvelles voies de communication entre des positions culturelles non dominantes. 


\section{Bibliographie}

\section{Bibliographie primaire}

Bâ, Ahmadou Hampaté. L'Étrange destin de Wangrin. Paris : 10/18, 1992.

Bugul, Ken. Riwan ou le chemin de sable. Paris : Présence africaine, 1999.

---. Riwan ali peščena pot. Trad. Katja Zakrajšek. Ljubljana : Sanje (à paraître en 2010).

Kane, Cheikh Hamidou. L'Aventure ambigüe. Paris : 10/18, 2002.

Kourouma, Ahmadou. Alabu ni treba. Trad. Katja Zakrajšek. Ljubljana : Sanje, 2004.

---. Allah n'est pas obligé. Paris : Seuil, 2000.

---. Les Soleils des indépendances. Paris : Seuil, 1970.

---. Monnè, outrages et défis. Paris : Seuil, 1990.

Nganang, Patrice. Temps de chien. Paris : Le Serpent à plumes, 2003.

Niane, Djibril Tamsir. Soundjata. Paris : Présence africaine, 1960.

---. Sundžata. Trad. Katja Zakrajšek. Ljubljana : Sanje, 2008-09.

Okara, Gabriel. The Voice. Londres : Heinemann, 1970.

Saro-Wiwa, Ken. Sozaboy. Londres : Longman, 1994.

---. Sozaboy: Pétit minitaire. Trad. Samuel Millogo et Amadou Bissiri. Paris : Actes sud, 1998.

---. Sozaboy. Trad. Andrej Skubic. Ljubljana : Beletrina, 2008.

\section{Bibliographie secondaire}

Appiah, Kwame Anthony. «Thick translation. » The Translation Studies Reader. Ed. Lawrence Venuti. Londres, New York : Routledge, 2000. 417-429.

Bakhtine, Mikhail. Esthétique et théorie du roman. Paris : Gallimard. 1978.

---. The Dialogic Imagination. Austin : University of Texas Press, 1981.

--- Teorija romana: iæ̧brane rąprave. Ljubljana : Cankarjeva založba, 1982.

Bandia, Paul F. Translation as Reparation : Writing and Translation in Postcolonial Africa. Manchester, Kinderhook : St. Jerome Publishing, 2008.

Batchelor, Kathryn. Decolonizing Translation : Francophone African Novels in English Translation. Manchester, Kinderhook : St. Jerome Publishing, 2009.

Bhabha, Homi K. The Location of Culture. Londres, New York : Routledge,1994.

Borgomano, Madeleine. Abmadou Kourouma : Le "guerrier" griot. Paris : L'Harmattan, 1998.

Caron, David. « Pour une poétique de l'opacité : Ahmadou Kourouma, Édouard Glissant et l'espace de la Relation francophone. » Revue canadienne de littérature comparée 25.3-4 (1998). 348-362.

Cordonnier, Jean-Louis. Traduction et culture. Paris : Hatier-Didier, 1995.

Gassama, Makhily. La Langue d'Ahmadou Kourouma ou le français sous le soleil d'Afrique. Paris : Karthala.

Gauvin, Lise. L'écrivain francophone à la croisée des langues : entretiens. Paris : Karthala, 1997.

Grutman, Rainier. Des langues qui résonnent: L'bétérolinguisme au XIXe siècle québécois. Montréal : Fides, 1997.

Inventaire des particularités lexicales du français en Afrique noire. Paris : EDICEF/AUPELF, 1988.

Lefebvre, Aurélie. « Patrice Nganang et "la parole des sous-quartiers" : une approche sociolinguistique de l'écriture du français dans le roman francophone. » Discours et écritures dans les sociétés en mutation: Itinéraires et contacts de cultures 39 (2007). 3547.

Martin, Patrice et Christophe Drevet. La langue française vue d'ailleurs. Casablanca : Tarik, 2001. 
Moro, Adriana. « La langue de la communication interculturelle. L'exemple de Les soleils des indépendances et de Monnè, outrages et défis d'Ahmadou Kourouma. » Littératures africaines : transpositions? Éd. Gilles Teulié. Montpellier : Université Paul-Valéry Montpellier III, 2002. str. 355- 368.

Pokorn, Nike K. « Kulturni materializem v teoriji prevajanja: Prevod kot kulturnopolitična dejavnost. » Literatura 167/168 (2005). 144-154.

Spivak, Gayatri Chakravorty. "The Politics of Translation. » The Translation Studies Reader. Ed. Lawrence Venuti. Londres, New York : Routledge, 2000. 397-416.

Tymoczko, Maria. Enlarging Translation, Empowering Translators. Manchester, Kinderhook : St. Jerome Publishing, 2007.

Venuti, Lawrence. «Translation, Community, Utopia. » The Translation Studies Reader. Ed. Lawrence Venuti. Londres, New York : Routledge, 2000. 468-488.

Vevar, Štefan. Temeljni aspekti in principi literarnega prevajanja. Ljubljana : Študentska založba, 2000.

Zabus, Chantal. «Othering the Foreign Language in the West-African Europhone Novel. » Revue canadienne de littérature comparée 17.3-4 (1990). 348-366.

Zakrajšek, Katja. «Texte postcolonial et sa traduction. » Discours et écritures dans les sociétés en mutation: Itinéraires et contacts de cultures 39 (2007). 181-193.

---. « Postkolonialni dialogi. » Primerjalna književnost 30/2 (2007). 19-34.

---. « K problematiki prevajanja afriškega evrofonskega romana v slovenščino. » Ars\& \&umanitas III/1-2 (2009). 157-172.

---. « Potujitveni in prisvajajoči prevod v luči medjezikovnih razmerij moči. » Hieronymus : Journal for Translation. 3.1-2 (2009). 7-20.

«Založništvo, Slovenija, 2008. » Statistični urad Republike Slovenije. Statistični urad Republike Slovenije, 2009. En ligne. Consulté le 8 sept. 2010. 